'Departamento de Cardiología. Clínica Universidad de los Andes. Santiago, Chile. ${ }^{2}$ Cardiologia 1. Dipartimento Cardiotoracovascolare, Gran Ospedale Metropolitano Niguarda. Milán, Italia. ${ }^{3}$ Clínica Alemana. Santiago, Chile. ${ }^{4}$ Departamento de Cardiología. Unidad de Cardiología Intervencional y Hemodinamia, Hospital Sótero del Río. Santiago, Chile.

Recibido el 25 de noviembre de 2019, aceptado el 15 de abril de 2020.

Correspondencia a: Dr. Nicolás Veas $\mathrm{P}$. Departamento de Cardiología. Clínica Universidad de los Andes. Avenida Plaza 2501. Las Condes. nicoveas@gmail.com

\section{Litoplastía iliaco-femoral para implante transcatéter de válvula aórtica}

\author{
NICOLÁS VEAS $S^{1,4}$, FRANCESCO SORIANO $^{2}$, JOSÉ WINTER R,4, \\ STEFANO NAVA $^{2}$, RENÉ HAMEAU ${ }^{4}$, DANTE LIDEFJELD ${ }^{1,4}$, \\ MARTÍN VALDEBENITO ${ }^{1,4}$, RODRIGO MUÑOZ $^{3,4}$, JACOPO OREGLIA $^{2}$
}

\section{Iliac-femoral lithoplasty for a transcatheter aortic valve implantation. Report of one case}

Transcatheter aortic valve implantation (TAVI) has a lower perioperative risk than traditional surgery mostly when the transfemoral access is used. Some patients have anatomical conditions that contraindicate the use of this route. Lithoplasty is a novel technique that fractures calcium in coronary and peripheral arteries using pulsatile waves transmitted through an angioplasty balloon. We report an 83 year-old male with an aortic stenosis requiring TAVI, with severe calcification of his femoral and aortic arteries. A balloon lithoplasty of the right iliac-femoral tract was carried out, which allowed the use of the transfemoral route to install the aortic prosthesis. The patient had a good subsequent evolution.

(Rev Med Chile 2020; 148: 548-552)

Key words: Peripheral Arterial Disease; Aortic Valve Stenosis; Transcatheter Aortic Valve Replacement.
E 1 implante transcatéter de válvula aórtica (TAVI) ha emergido como una alternativa terapéutica válida en pacientes con estenosis aórtica sintomática ${ }^{1-2}$ e incluso en pacientes de bajo riesgo ${ }^{3}$. Los resultados favorables en términos de morbimortalidad están dados principalmente por el uso del acceso femoral en comparación a un acceso alternativo ${ }^{4}$.

A pesar del avance tecnológico, que ha logrado mejorar el perfil de cruce de las distintas TAVI, es frecuente encontrar pacientes con accesos complejos, ya sea por diámetro o por marcada calcificación en el trayecto iliaco-femoral. La presencia de calcificación arterial periférica aumenta la complejidad en los tratamientos percutáneos y las complicaciones relacionadas al procedimiento ${ }^{5}$.

La litotripsia es un tratamiento que se ha usado tradicionalmente para la fragmentación de cálculos renales, actuando por medio de una onda de sonido de alto poder que atraviesa el tejido conectivo y selectivamente interactúa con el calcio. Gracias a avances en esta técnica, se ha logrado adaptar para el manejo de las lesiones cálcicas en otros territorios, como el vascular periférico y, posteriormente, en las arterias coronarias. Esta técnica, llamada litoplastía, ha sido introducida como una nueva opción terapéutica en pacientes con importante calcificación en estos territorios ${ }^{5-7}$. Su mecanismo de acción consiste en la emisión de ondas pulsátiles a través de un balón de angioplastia, logrando la disrupción y fragmentación del calcio, disminuyendo el daño vascular. En pacientes candidatos a TAVI, este avance ha permitido mantener el uso del acceso femoral en sujetos con anatomías intensamente calcificadas, manteniendo, por ende, el beneficio de su uso sin aumentar el riesgo y potenciales complicaciones vasculares.

A continuación, presentamos un caso clínico de un paciente con enfermedad de 3 vasos y estenosis aórtica grave sintomática, donde se realiza angioplastia (PCI) multivaso e implante de TAVI por vía femoral, luego de litoplastía iliaco-femoral. 


\section{Caso clínico}

Paciente masculino de 83 años, con antecedentes de hipertensión arterial, diabetes mellitus tipo 2 , enfermedad renal crónica etapa 3 , enfermedad pulmonar obstructiva crónica y estenosis aórtica de grado moderado en seguimiento. Sin historia de claudicación.

Ingresó a servicio de urgencia por cuadro de angina de reposo, asociado a cambios en el electrocardiograma en pared lateral. El ecocardiograma superficie (ETT) mostraba alteraciones segmentarias en el mismo territorio, con fracción de eyección normal. Había progresión de la estenosis aórtica en una válvula de tres velos con gradiente valvular medio de $46 \mathrm{mmHg}$, velocidad máxima de $4,5 \mathrm{~m} / \mathrm{sec}$ y un área valvular calculada de $0,8 \mathrm{~cm}^{2}$. Evolucionó con alza de troponina, por lo que se decidió estudio coronario. En la coronariografía se observó enfermedad calcificada y grave de 3 vasos. La evaluación con tomografía computada (TAC) mostraba la aorta gravemente calcificada, con mediciones que sugerían el uso de una válvula Evolut $^{\mathrm{TM}}$ Pro\#26 (Medtronic, Fridley, MN, USA), en caso de TAVI. Los accesos femorales estaban marcadamente calcificados, sobre todo a nivel de iliacas comunes, donde el diámetro mínimo (MLD) era de 5,2 x 3,6 mm a derecha, con calcificación prácticamente en 360 grados (Figura 1). Esto último dificultaría el avance de un sistema de entrega 16F necesario para desplegar esta prótesis.

La evaluación también concluyó un alto riesgo quirúrgico (score STS 12,3\% y Euroscore II $10,23 \%)$. Evaluado por el equipo de especialistas se planteó una resolución percutánea. Descartamos accesos alternativos, dada la fragilidad del paciente y no era candidato a denudación arterial, puesto que el principal problema era a nivel iliaco. Se planteó en primer lugar angioplastia multivaso $y$, posteriormente, un implante de TAVI transfe-

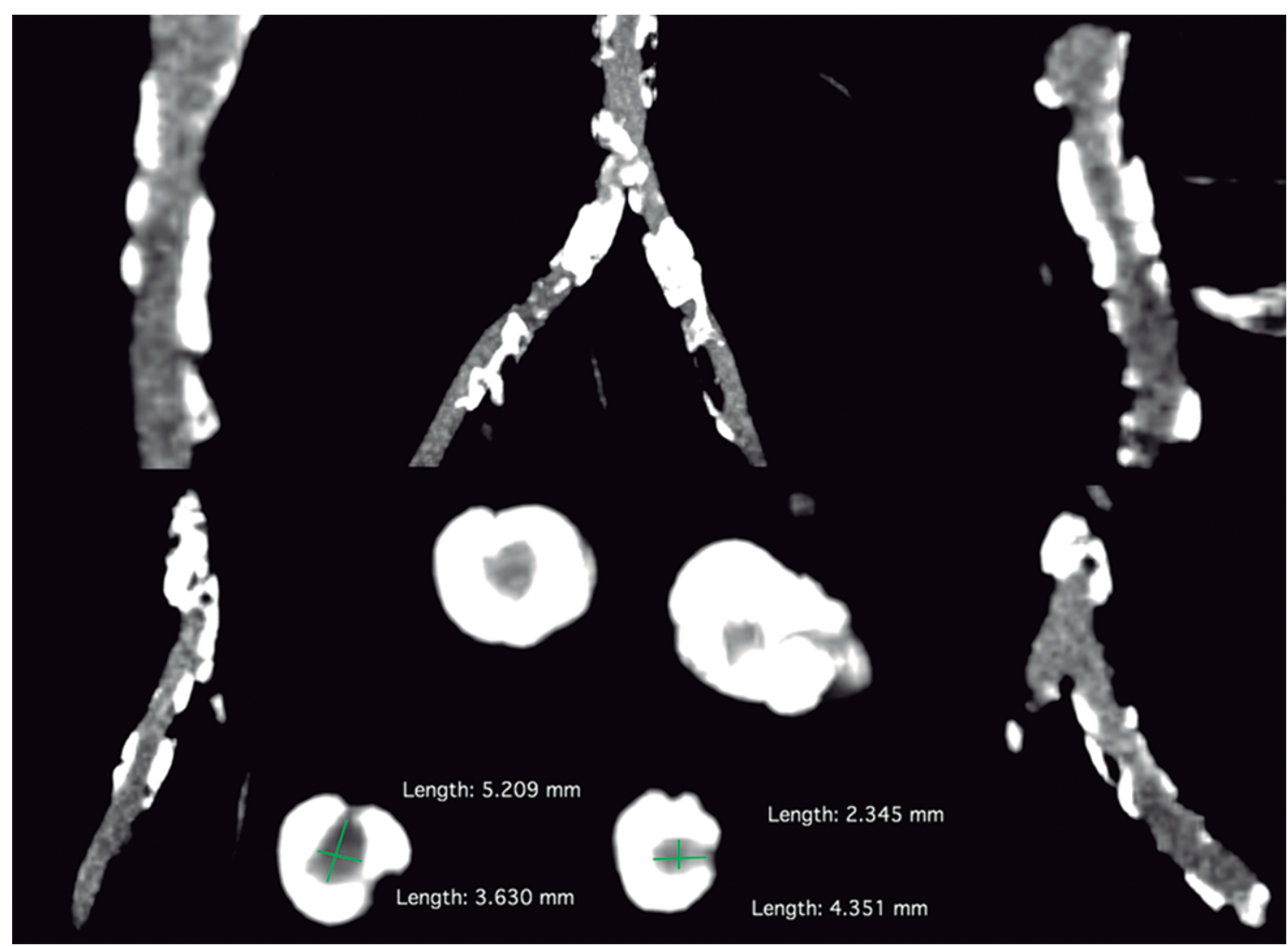

Figura 1. AngioTAC con protocolo TAVI que muestra calcio en tracto aorto-iliaco-femoral. Se evidencia calcio circunferencial con diámetros de 5,2 × 3,6 mm a derecha y de 2,3 × 4,3 mm a izquierda en sus diámetros más estrechos. 
moral con litoplastía iliaco-femoral derecha con técnica minimalista.

Se realizó angioplastia vía arteria radial derecha con catéter $7 \mathrm{~F}$ guiada con ultrasonido intravascular (IVUS). Se utilizó Rotablator ${ }^{\mathrm{TM}}$ (Boston Scientific, Malborough, MA, USA) en los 3 vasos, dada la calcificación circunferencial, e implante de stents medicados, sin incidentes. Evolucionó favorablemente, sin alza significativa de creatinina.

Dejamos el acceso femoral derecho para el implante de la TAVI y el femoral izquierdo para protección e inyecciones contralaterales. Punción eco-guiado de ambas femorales. Protección contralateral con guía periférica V-18 (Boston Scientific). Obtuvimos imágenes IVUS que nos mostraban un MLD de 4,5 x 4,3 $\mathrm{mm}$ a nivel de iliaca común derecha con calcio circunferencial importante (Figura 2A-2B). Optamos realizar litoplastía (ShockWave Medical, Santa Clara, CA,
USA) con balón de 7 x $60 \mathrm{~mm}$ insuflado a 4 atm y luego a 8 atm por 30 segundos, cada uno según protocolo (Figura 2C), con 5 fases de 30 pulsos en todo el trayecto iliaco femoral derecho. Procedimiento sin incidentes, sin imágenes de disección angiográficas ni al IVUS. Luego de ello se avanzó el introductor Inline con válvula Evolut Pro \#26 sin dificultad (Figura 2D). Posteriormente, se implantó exitosamente la bioprótesis aórtica, sin leak paravalvular y con buen resultado hemodinámico (Figura 2E). Se retiró el sistema de entrega y se intercambió por un introductor Sentrant $16 \mathrm{~F}$. El cierre final del acceso femoral derecho se realizó con Perclose ProGlide ${ }^{\mathrm{TM}}$ Suture-Mediated Closure (Abbott Laboratories, Chicago, IL, USA) implantados previamente. No se evidenciaron complicaciones en los trayectos iliaco-femorales derechos (Figura 2F). Finalizando con cierre de acceso femoral izquierdo.
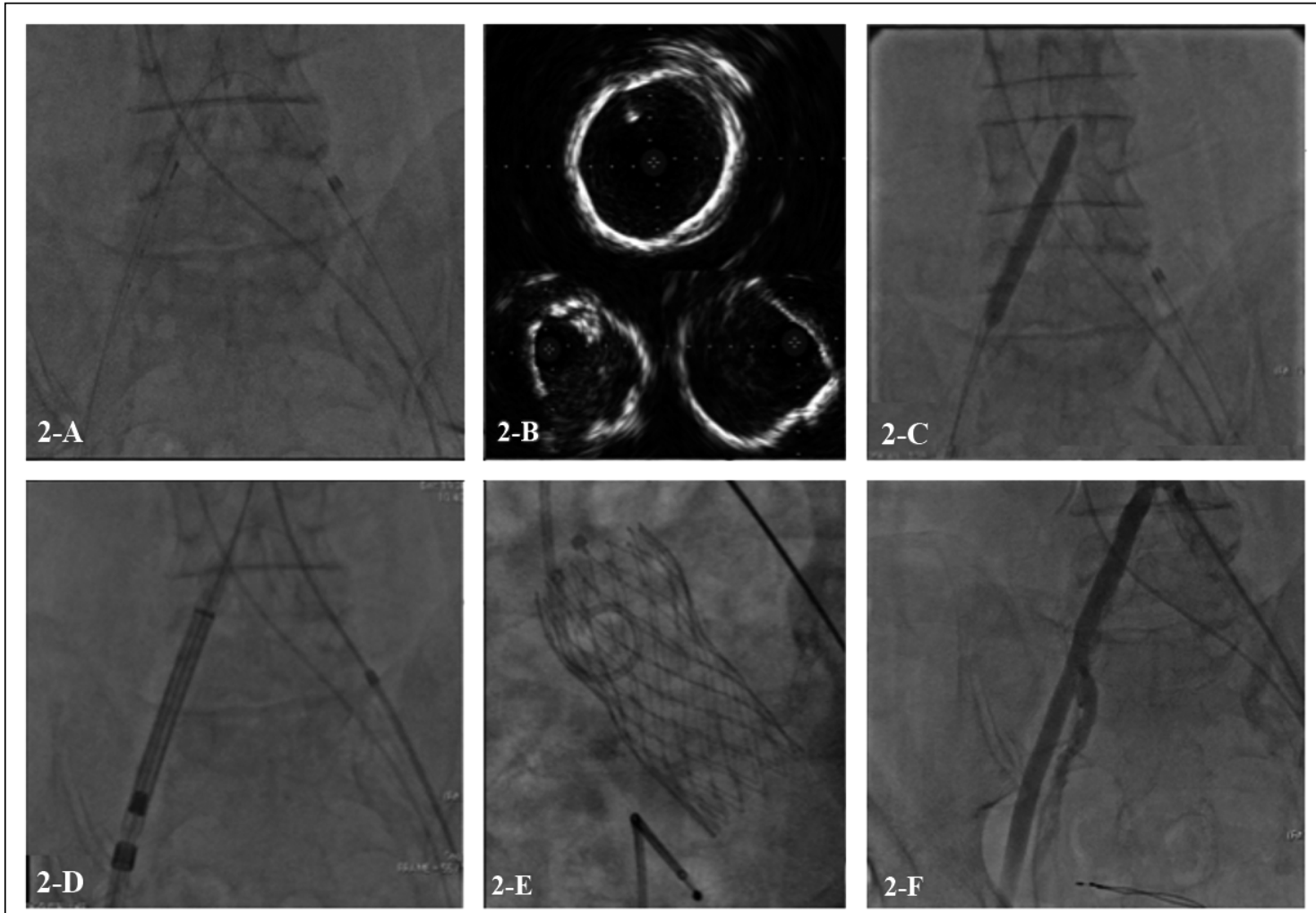

Figura 2. A: Angiografía que muestra guía V-18 de protección en femoral derecha y paso de sonda IVUS. B: Imágenes IVUS a nivel iliaco-femoral en distintos puntos, demostrando calcio circunferencial, con diámetro mínimo por esta técnica de 4,5 x 4,3 mm. C: Momento de insuflación de sistema de litoplastía (Shockwave) balón de 7 x $60 \mathrm{~mm}$. D: Paso de introductor Inline Sheat sin dificultad. E: TAVI Evolut Medronic en posición aórtica. F: Angiografía final, descartando complicaciones posimplante de TAVI. 
El paciente evolucionó favorablemente, con un seguimiento a 9 meses sin eventos clínicos ni complicaciones vasculares periféricas. El paciente autorizó la publicación de este caso clínico.

\section{Discusión}

La calcificación vascular es un problema importante en la cardiología intervencional. Esta aumenta la complejidad y conlleva a una mayor proporción de complicaciones, tanto a nivel coronario como periférico 5 . El hallazgo de calcio a nivel vascular se ha vuelto frecuente debido al envejecimiento de la población, por lo que es necesario estar familiarizados con los distintos dispositivos que nos permitan su manejo, ya sea modificando o fracturando las placas calcificadas.

El grupo que presenta un mayor beneficio en TAVI con respecto a morbimortalidad son aquellos pacientes donde se utiliza el acceso femoral. Lo anterior confiere una gran importancia a su uso de forma segura y sin complicaciones. Hasta hoy no existe un acceso alternativo (subclavio, carotídeo, transaórtico o transcavo) que logre los resultados de la vía transfemoral ${ }^{4}$. La ocurrencia de una complicación vascular mayor aumenta la mortalidad considerablemente, pudiendo llegar incluso hasta $50 \%$ en pacientes con uso de anticoagulantes ${ }^{6}$. El principal predictor de complicación es el diámetro del trayecto iliaco-femoral, dado que existe una correlación entre el diámetro del introductor y el diámetro de la arteria con las posibles complicaciones vasculares. Con un punto de corte de esta relación en 1,05 . Sobre este punto existe un aumento de las complicaciones vasculares mayores $(30,9 \%$ vs $5,6 \%, \mathrm{p}<0,001)$ y de la mortalidad a 30 días $(18,2 \% \text { vs } 2,8 \%, p<0,004)^{7}$.

La litoplastía es una técnica basada en la litotripsia renal, donde se entregan ondas de pulsos emitidas a través de un balón de angioplastía, permitiendo la disrupción del calcio tanto superficial como el profundo ${ }^{8-9}$. El estudio DISRUPT PAD ${ }^{10}$ evaluó de forma prospectiva, no aleatoria a 95 pacientes sometidos a litoplastía en la arteria femoral superficial y en la poplítea. Se observó un aumento inmediato de al menos $3 \mathrm{~mm}$ en el diámetro de la arteria, con 96,8\% de éxito en la revascularización del vaso objetivo y menos de $25 \%$ de estenosis, residual con baja tasa de implante de stent ${ }^{10}$. Esto, sin aumentar las complicaciones vasculares como disecciones o ruptura del vaso. A nivel coronario, pese a un favorable perfil de seguridad, ya existen reportes de complicaciones con disecciones producto de ruptura del balón de litoplastía ${ }^{11}$.

En este paciente, el uso de esta tecnología permitió utilizar la vía transfemoral en un paciente con contraindicación inicial para el uso de este acceso. Realizándose sin complicaciones y permitiendo el implante exitoso de la válvula. Se han publicado casos clínicos similares, en donde el uso de esta técnica permitió el implante de TAVI o el posicionamiento de asistencia ventricular percutánea como Impella que requiere mayor diámetro femoral para el paso de estos dispositivos ${ }^{12-14}$. Aun así, se necesitan mayores estudios de distribución aleatoria para confirmar la eficacia y la seguridad de esta técnica, la cual tiene resultados iniciales promisorios.

\section{Referencias}

1. Leon MB, Smith CR, Mack MJ, Makkar RR, Svensson LG, Kodali SK, et al. Transcatheter or surgical aortic-valve replacement in intermediate-risk patients. N Engl J Med. 2016; 374: 1609-20.

2. Mack MJ, Leon MB, Smith CR, Miller DC, Moses JW, Tuzcu EM, et al. 5-year outcomes of transcatheter aortic valve replacement or surgical aortic valve replacement for high surgical risk patients with aortic stenosis (PARTNER 1): A randomized controlled trial. Lancet. 2015; 385: 2477-84.

3. Mack MJ, Leon MB, Thourani VH, Makkar R, Kodali SK, Russo M, et al. Transcatheter Aortic-Valve Replacement with a Balloon-Expandable Valve in Low-Risk Patients. N Engl J Med. 2019; 380 (18): 1695-705.

4. Doshi R, Shah P, Meraj PM. In-hospital outcomes comparison of trans-femoral vs transapical transcatheter aortic valve replacement in propensity-matched cohorts with severe aortic stenosis. Clin Cardiol. 2018; 41: 32632.

5. Généreux P, Madhavan MV, Mintz GS, Maehara A, Palmerini T, Lasalle L, et al. Ischemic outcomes after coronary intervention of calcified vessels in acute coronary syndromes. Pooled analysis from the HORIZONS-AMI (harmonizing outcomes with revascularization and stents in acute myocardial infarction) and ACUITY (acute catheterization and urgent intervention triage strategy) TRIALS. J Am Coll Cardiol. 2014; 63 (18): 1845-54.

6. Généreux P, Cohen DJ, Mack M, Rodes-Cabau J, Yadav 
M, Xu K, Parvataneni R, Hahn R, Kodali SK, Webb JG, Leon MB. Incidence, predictors, and prognostic impact of late bleeding complications after transcatheter aortic valve replacement. J Am Coll Cardiol 2014; 64: 2605-15.

7. Hayashida $K$, Lefèvre $T$, Chevalier $B$, Hovasse $T$, Romano M, Garot P, Mylotte D, Uribe J, Farge A, Donzeau-Gouge P, Bouvier E, Cormier B, Morice MC. Transfemoral aortic valve implantation new criteria to predict vascular complications. JACC Cardiovasc Interv 2011; 4: 851-8.

8. Brinton TJ, Brodmann M, Werner M, Tepe G, Holden A, Scheinert D, Wolf F, et al. Safety and performance of the Shockwave Medical Lithoplasty System in treating calcified peripheral vascular lesions: 6-month results from the two-phase DISRUPT PAD study. J Am Coll Cardiol. 2016; 68 (18S): B314.

9. Brinton TJ, Hill J, Ali Z. DISRUPT CAD: A multicentre, prospective, single-arm study of percutaneous lithoplasty prior to stent implantation in heavily calcified coronary lesion. J Am Coll Cardiol 2016; 18 S (TCT Suppl): 68.

10. Zeller T. Impact of lithoplasty: Disrupt PAD. Abstract presented at: VIVA 2016; September 18-22, 2016; Las Vegas, NV, USA; 2016.

11. Soriano F, Veas N, Piccinelli E, Oreglia J. Coronary dissection due to intravascular lithoplasty balloon rupture.
EuroIntervention. 2019; 15 (6): e558-9.

12. Riley RF, Corl JD, Kereiakes DJ. Intravascular lithotripsy-assisted Impella insertion: A case report. Catheter Cardiovasc Interv. 2019; 93 (7): 1317-9.

13. Gorla R, Cannone GS, BedogniF, De Marco F. Transfemoral aortic valve implantation following lithoplasty of iliac artery in a patient with poorvascular access. Catheter Cardiovasc Interv. 2019; 93 (3): E140-E142.

14. López D, Sanmartín X, Trillo R, Cid B, Antúnez P, González J. Shockwave Lithoplasty-facilitated transfemoral access for transcatheter aortic valve replacement. An initial single-center experience in Spain. Rev Esp Cardiol. 2019; 72 (11): 980-2.

15. Brodmann M, Werner M, Britton T, et al. Safety and performance of Lithoplasty for treatment of calcified peripheral artery lesions. JACCLett. 2017; 70 (7): 908-9.

16. Babaev A, Zavlunova S, Attubato MJ, Martinsen BJ, Mintz GS, Maehara A. Orbital atherectomy plaque modification assessment of the femoropopliteal artery via intravascular ultrasound (TRUTH study). Vasc Endovas Surg. 2015; 49 (7): 188-94.

17. Ali ZA, Brinton TJ, Hill JM, Maehara A, Matsumura M, Karimi Galougahi K, et al. Optical coherence tomography characterization of coronary lithoplasty for treatment of calcified lesions: First description. JACC Cardiovasc Imaging. 2017; 10: 897-906. 